\title{
Peertechz
}

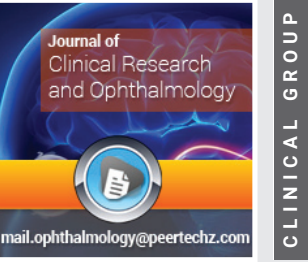

\section{Analysis of Effectiveness of}

\section{Treatment of Meibomian}

\section{Gland Dysfunction in Patients} with Computer Vision

\section{Syndrome}

\author{
Kavitha Chikkanayaknahalli Venugopal ${ }^{1}$, Pavana Acharya ${ }^{2}$, \\ Shruthi Kavinamoole ${ }^{3 *}$ and Spoorti Mundinamani ${ }^{3}$ \\ ${ }^{1}$ Professor, Department of Ophthalmology, Hassan Institute of Medical Sciences, Hassan, Karnataka, \\ India \\ ${ }^{2}$ Assistant Professor, Department of Ophthalmology, Hassan Institute of Medical Sciences, Hassan, \\ Karnataka, India \\ ${ }^{3}$ Postgraduate Student, Department of Ophthalmology, Hassan Institute of Medical Sciences, Hassan, \\ Karnataka, India
}

Received: 11 January, 2021

Accepted: 02 February, 2021

Published: 03 February, 2021

*Corresponding authors: Shruthi Kavinamoole, Postgraduate Student, Department of Ophthalmology, Hassan Institute of Medical Sciences, Room No.606, HIMS Ladies Hostel, HIMS, Hassan, Karnataka, India, Tel: 9483621812; E-mail: shruthik2393@gmail.com

Keywords: Meibomian gland dysfunction; Computer vision syndrome; Tear supplements; Azithromycin eye ointment

https://www.peertechz.com

Check for updates

\section{Abstract}

Aims: To evaluate association, stage and treatment efficacy of treating Meibomian Gland Dysfunction(MGD) in Computer Vision Syndrome(CVS) patients.

Setting: Department of ophthalmology, Hassan Institute of Medical Sciences, Hassan, Karnataka, India.

Design: Prospective observational study.

Materials and methods: Prospective study on 232 CVS patients were evaluated for MGD and randomly divided into groupA and B. GroupA were treated with tear supplements. GroupB were treated with azithromycin ointment and tear supplements. Efficacy was analysed at end of $3 \mathrm{months.}$

Statistical analysis: Data was analysed using descriptive statistics.

Results: Among 232 CVS patients, MGD was found in 90(38.8\%) patients. Among them, 38had stage1, 36had stage2, 14 had stage 3 and 2 patients had stage4 MGD. By the end of three months, in groupA, 34of 48 patients with stage 1 and $2 \mathrm{MGD}$ were cured. In groupB, 36 of 42 patients with all stages of MGD were cured. $100 \%$ of the CVS patients with stage $1,66.7 \%$ of stage 2 and $50 \%$ of the stage 3 MGD improved completely with tear supplements alone whereas $25 \%$ of stage 3 MGD patients improved to stage 1 and $25 \%$ of stage 3 MGD did not improve at the end of three months. But, $100 \%$ of the CVS patients with stage $1,77.8 \%$ of stage $2,100 \%$ of stage 3 improved completely and 1patient with stage4 MGD improved to stage1 by the end when treated with tear supplements and azithromycin ointment.

Conclusion: $39 \%$ of CVS patients have MGD. So while treating CVS patient, its important to evaluate and treat MGD too. Tear supplements alone can be considered as a treatment option for CVS patients with stage 1 and 2MGD whereas stage3 and 4MGD needs azithromycin ointment in addition to tear supplements for a good patient outcome. 


\section{Introduction}

In the recent past, there has been a large growth in the field of technology with the use of computers and smart phones etc. The introduction of computer no doubts has revolutionized and benefited the society; however it is associated with healthrelated problems which includes computer vision syndrome $[1,2]$. The American Optometric Association defines computer vision syndrome as a complex of eye and vision problems related to the activities which stress the near vision and which are experienced in relation, or during, the use of the computer [3]. Computer vision syndrome mainly occurs because of three major mechanisms-1)extraocular 2)ocular surface 3) accommodative mechanisms [4]. Ocular symptoms includes dryness, irritation, burning sensation in the eyes, visual symptoms like blurring, or double vision and musculoskeletal symptoms including neck and shoulder pain. The major factors associated with CVS are either environmental, improper lighting, display position and viewing distance and/or dependent on the user's visual abilities uncorrected refractive error, oculomotor disorders and tear film abnormalities [4].

The meibomian glands are the glands present in the tarsal plate that secrete lipid portion of the tear film which helps in maintaining the moisture content in the anterior segment of the eye. Meibominitis is the inflammation of meibomian glands leading to meibomian gland dysfunction where the meibomian glands do not secrete enough lipid or the quality of the lipid is not good [5].

Computer vision syndrome can cause symptoms similar to the symptoms due to meibomian gland dysfunction $[6,7]$.

CVS has a multifactorial causation [8]. Meibomian gland dysfunction due to meibominitis is one among them. Hence, we aimed at studying the efficacy of treating meibomian gland dysfunction in patients with CVS.

\section{Objectives of the study}

a. To evaluate the association of meibomian gland dysfunction in patients with computer vision syndrome

b. To assess the grade of meibomian gland dysfunction in patients with computer vision syndrome

c. To assess the efficacy of treatment of meibomian gland dysfunction in patients with computer vision syndrome.

\section{Materials and methods}

A prospective observational multicentric study was conducted in about 232 attending out patient Department of Ophthalmology at HIMS Hassan and Vasan Eye care hospital, Hassan during the study period 1/02/2018 to 31/07/2018. The patients were included in the study by applying the following Inclusion and Exclusion criteria. Institutional Ethics committee-HIMS, Hassan- clearance obtained for the study.

\section{Inclusion criteria}

Patients belonging to either sex irrespective of age diagnosed to have computer vision syndrome attending outpatient department, HIMS teaching hospital, Hassan and Vasan Eye care hospital, Hassan were included in the study.

\section{Exclusion criteria}

Following patients were excluded from the study-

1. Patients with diabetes mellitus

2. Patients with autoimmune causes of dry eye like primary Sjogren's syndrome,

3. Patients with glaucoma

4. Patients on topical steroids

5. Patients with other ocular infections.

\section{Methodology}

Patients fulfilling the inclusion criteria were recruited into the study.

The aims and objectives of the intended study was properly explained to the subjects and informed consent was taken. Data was collected as per the proforma sheet.

Ophthalmological workup including Visual acuity, slit lamp bio microscopy, intraocular pressure measurement using applanation tonometry was done in all patients.

Patients diagnosed with computer vision syndrome based on the following criteria $[7,8]$.

> Symptoms like eyestrain, burning sensations, irritation, redness, blurred vision, and dry eyes

$>$ History of visual display units usage.

These patients were subjected for detailed anterior segment evaluation and looked for the presence of meibomian gland dysfunction.

- Following tests were done to these patients - Lid margin examination under slit lamp to look for pouting, drop outs, erythema of lid margin

- Tear film breakup time,

- Schirmer's test,

- Meibomian gland secretions and expressiblity

- Ocular surface staining using Rose Bengal stain.

The patients with meibomian gland dysfunction were divided into stage 1, 2, 3 and 4 as follows-[9]

\section{Stage 1}

a. No symptoms of ocular discomfort, itching or photophobia

b. Clinical signs of MGD based on gland expression minimally altered secretions: Grade $>2$ to $<4$ 


\section{Expressibility: 1}

c. No ocular surface staining.

\section{Stage 2}

a. Minimal to mild symptoms - ocular discomfort, itching or photophobia

b. Minimal to mild MGD clinical signs- scattered lid margin features mildly altered secretions: Grade $>4$ to $<8$

\section{Expressibility: 1}

c. None to limited ocular surface staining (DEWS grade 0-7; Oxford grade $0-3$ ).

\section{Stage 3}

a. Moderate symptoms of ocular discomfort, itching or photophobia with limitations of activities

b. Moderate MGD clinical signs - lid margin features: plugging, vascularity moderately altered secretions: Grade $>8$ to $<13$

Expressibility: 2

c. Mild to moderate conjunctival and peripheral corneal staining, often inferior (DEWS grade 8-23; Oxford grade $4-10)$.

\section{Stage 4}

a. Marked symptoms of ocular discomfort, itching or photophobia with definite limitations of activities

b. Severe MGD clinical signs increased lid margin features: dropout, displacement severely altered secretions: Grade $>13$ Expressibility: 3

c. Increased conjunctival and corneal staining, including central staining (DEWS grade 24-33; Oxford grade 11-15).

d. Increased Signs of inflammation: e.g., > moderate conjunctival hyperemia, phlyctenules [9].

Meibum quality was assessed in each of 8 glands of the central third of the lower lid on a $0-3$ scale for each gland: $0=$ clear meibum; $1=$ cloudy meibum; $2=$ cloudy with debris (granular); 3=thick, like toothpaste (range 0-24).

Expressibility of meibum was assessed from 5 glands: $0=$ all glands expressible; $1=3-4$ glands expressible; $2=1-2$ glands expressible; $3=$ no glands expressible. This can be assessed in the lower or upper lid [9].

These patients with MGD were randomly divided into group A and group B . Patients in group A were treated with artificial tear drops alone. Group B patients were treated with azithromycin eye ointment and artificial tear drops.
Patients were followed up at the end of 1 month and 3months. Both the group patients were assessed based on -

1. Subjective improvement

2. Schirmer's test

3. Tear film breakup time

4. Presence of tear film debris, secretions

5. Meibomian gland expressibility

6. Ocular surface staining with Rose Bengal

Outcome of the two groups were compared and efficacy of treating computer vision syndrome patients for meibomian gland dysfunction was analysed.

\section{Statistical analysis}

Data was entered in Microsoft Excel and analyzed using SPSS version 22 software. Descriptive statistical analysis was done. The results were expressed in frequencies, percentage, means, and standard deviations.

Chi square test was used for categorical variables. Results with $\mathrm{P}$ value $<0.05$ were considered as Significant.

\section{Results}

Out of 232 patients with computer vision syndrome(CVS), 120(51.7\%) were males, and $112(48.3 \%)$ were females (Figure 1). The mean age was 24.00 years with a range of 15 years to 42 years.

Meibomian gland dysfunction (MGD) was found only in 90 $(38.8 \%)$ patients . $142(61.2 \%)$ patients did not have evidence of meibomian gland dysfunction(MGD). Among them, 38(16.4\%) had stage 1 MGD, 36(15.5\%) had stage 2 MGD, 14(6.0\%) had stage 3 and two patients(0.9\%) had stage 4 MGD (Figure 2).

$165(71.6 \%)$ patients were short term(<4hrs/day) VDU users and $67(28.4 \%)$ were long term ( $\geq 4 \mathrm{hrs} /$ day) VDU users. $228(98.3 \%)$ of the patients had a visual acuity of better than $6 / 12.170(73.3 \%)$ of them belonged to upper middle, $47(19.8 \%)$ to lower middle and $15(6.9 \%)$ belonged to upper class of Kuppuswamy class of socioeconomic strata.

Among these 232patients, 94(40.5\%) patients had a reduced blink rate $(<10 / \mathrm{min}) .118(50.9 \%)$ had blink rate range of $10-$

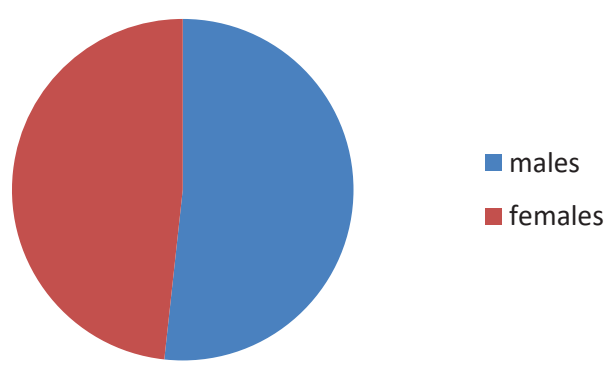

Figure 1: Gender distribution- among 232 patients with computer vision syndrome(CVS), 120(51.7\%) were males, and $112(48.3 \%)$ were females.

Citation: Venugopal KC, Acharya P, Kavinamoole S, Mundinamani S (2021) Analysis of Effectiveness of Treatment of Meibomian Gland Dysfunction in Patients with Computer Vision Syndrome. J Clin Res Ophthalmol 8(1): 001-006. DOI: https://dx.doi.org/10.17352/2455-1414.000084 
$15 /$ min and only $20(8.6 \%)$ had blink rate $>15 /$ min. Tear film breakup time(TBUT) was found to be markedly reduced $<5$ secs in $94(40.5 \%)$ patients. $112(48.3 \%)$ had TBUT between $6-10$ sec, $26(11.2 \%)$ patients had normal TBUT of more than 10 secs. $184(79.3 \%)$ patients had normal schirmer's test $(>10 \mathrm{~mm})$, only $10(4.3 \%)$ had severe dry eye with $<5 \mathrm{~mm}$ schirmer's test at the end of five minutes.

Among the short term VDU users, 16(9.6\%) patients had stage 1 meibomian gland dysfunction (MGD), 26(15.7\%) had stage 2 MGD. Among the long term VDU users, 22 patients (33.3\%) had stage $1 \mathrm{MGD}, 10(15.2 \%)$ had stage 2 MGD. This difference has been found statistically significant $(\mathrm{p}<0.001)$ (Figure 3). MGD was seen in $50(55.6 \%)$ patients using VDU $<1$ year, and $30(33.3 \%)$ patients using VDU for $1-5$ years $(\mathrm{p}=0.11)$.

Among the patients without MGD, only 26(18.3\%) had a blink rate $<10 / \mathrm{min}$, where as, $78(75.6 \%)$ patients with MGD had significant reduction in blink rate $<10 / \mathrm{min}$ (Table 1 ). Among the CVS patients with MGD, 10 (11.1\%) patients had TBUT<5sec, and no $(0 \%)$ patients had TBUT $>10$ secs. Where as CVS patients without MGD, 84 (59.2\%) had TBUT <5secs, 26(28.9\%) had TBUT $>10$ secs. This difference was statistically significant. $(\mathrm{p}<0.001)$ (Table 2).

All the patients without MGD had schirmer's test $>10 \mathrm{~mm}$ but $42(46.7 \%)$ patients with MGD had schirmer's $>10 \mathrm{~mm}$, $19(42.2 \%)$ had schirmer's $6-10 \mathrm{~mm}$ and 10 patients $(11.1 \%)$ had schirmer's $<5 \mathrm{~mm}(\mathrm{p}<0.001)$. (Table 3$)$. This difference was found to be statistically significant.

Among the VDU users <4hrs/day, 58(34.9\%) had a reduced blink rate $<10 / \mathrm{min}$, but $36(54.5 \%)$ patients who where using $\mathrm{VDU} \geq 4 \mathrm{hrs} /$ day had a reduced blink rate $(\mathrm{p}=0.125)$ (Table 4). 12(7.2\%) of VDU users $>4$ hrs/day had TBUT $<5$ secs and $14(21.2 \%)$ of VDU users $\geq 4$ hrs/day had TBUT $<5$ secs $(\mathrm{p}=0.027)$ (Table 5).

Among the 90 patients with MGD, 48 of them were put to group A and 42 to group B. Among this 48 patients in group A, $22(45.6 \%)$ had stage $1 \mathrm{MGD}, 18(37.5 \%)$ patients had stage 2 MGD,8(16.7\%)had stage 3 MGD. Among 42 patients in group B, $16(38.1 \%)$ had stage 1 MGD, $18(42.9 \%)$ had stage 2 MGD, $6(14.2 \%)$ had stage $3 \mathrm{MGD}$, two $(4.8 \%)$ patients had stage 4 MGD (p<0.007) (Table 6).

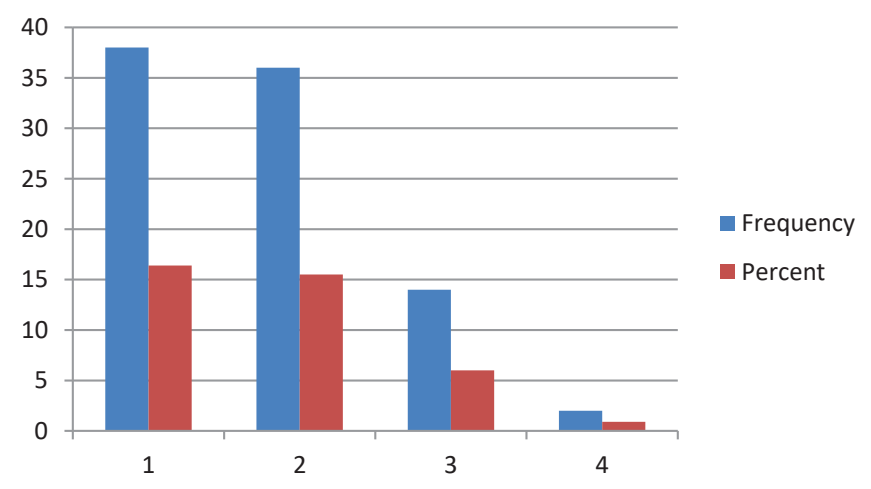

Figure 2: Stage of meibominitis at the time of enrollment $38(16.4 \%)$ had stage MGD, 36(15.5\%) had stage 2 MGD, $14(6.0 \%)$ had stage 3 and two patients( $0.9 \%)$ had stage 4 MGD.

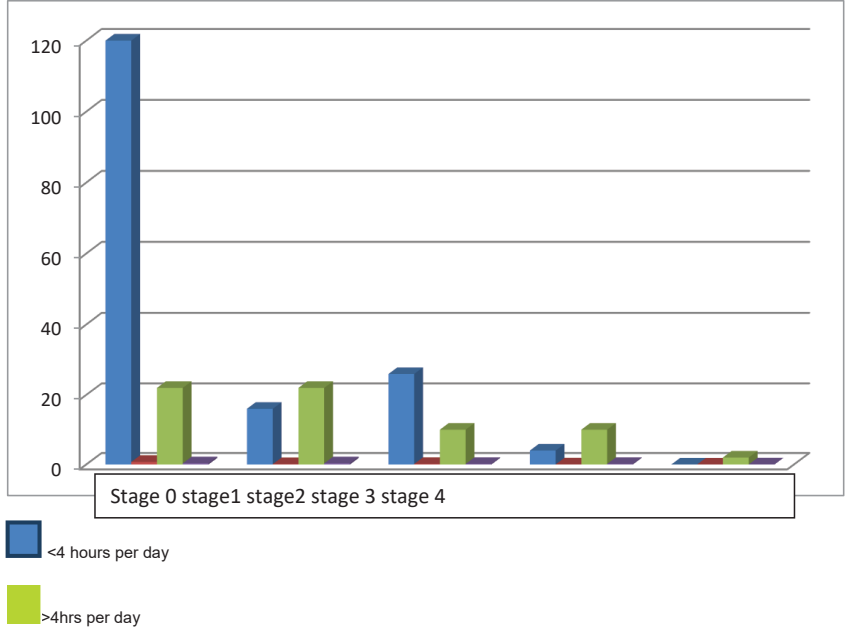

Figure 3: Number of hours of VDU usage per day. Among the short term $(<4 \mathrm{hrs}$ day) VDU users, 16(9.6\%) patients had stage 1 meibomian gland dysfunction (MGD), 26(15.7\%) had stage 2 MGD, 4(2.4\%) had stage 3. Among the long term VDU users(>4hrs/day), 22 patients (33.3\%) had stage 1 MGD, $10(15.2 \%)$ had stage 2 MGD, $10(15.2 \%)$ had stage 3 MGD and 2(3\%) hd stage 4 MGD

Table 1: Blink rate in patients with and without MGD.- Among the patients without MGD, only $26(18.3 \%)$ had a blink rate $<10 / \mathrm{min}$, where as, $78(75.6 \%)$ patients with MGD had significant reduction in blink rate $<10 / \mathrm{min}$.

\begin{tabular}{|c|c|c|c|c|}
\hline & \multicolumn{3}{|c|}{ Blinkrate } & \multirow{2}{*}{ Total } \\
\hline \multirow{2}{*}{ No MGD } & $\mathbf{1 5 / \mathbf { m i n }}$ & $\mathbf{1 0 - 1 5 / m i n}$ & $<\mathbf{1 0 / m i n}$ & \\
\hline \multirow{2}{*}{ MGD present } & 20 & 96 & 26 & 142 \\
\hline & $14.1 \%$ & $67.6 \%$ & $18.3 \%$ & $100.0 \%$ \\
\hline Tota & 0 & 22 & 68 & 90 \\
\hline & 20 & $24.4 \%$ & $75.6 \%$ & $100.0 \%$ \\
\hline & $8.6 \%$ & $50.9 \%$ & $40.5 \%$ & $100.0 \%$ \\
\hline
\end{tabular}

Table 2: Tear film break up time in patients with and without MGD- Among the CVS patients with MGD, $10(11.1 \%)$ patients had TBUT<5sec, and no $(0 \%)$ patients had TBUT>10secs. Where as CVS patients without MGD, 84 (59.2\%) had TBUT <5secs, 26(28.9\%) had TBUT $>10$ secs. This difference was statistically significant.

\begin{tabular}{|c|c|c|c|c|}
\hline & \multicolumn{3}{|c|}{ TBUT } & \multirow{2}{*}{ Total } \\
\hline \multirow{2}{*}{ No MGD } & $<\mathbf{5 s e c}$ & $\mathbf{5 - 1 0 s e c}$ & $>\mathbf{1 0 s e c}$ & 142 \\
\hline \multirow{2}{*}{ MGD present } & 84 & 58 & 0 & $100.0 \%$ \\
\hline & $59.2 \%$ & $40.8 \%$ & $.0 \%$ & 90 \\
\hline Total & 10 & 54 & 26 & $100.0 \%$ \\
\hline & $11.1 \%$ & $60.0 \%$ & $28.9 \%$ & 232 \\
\hline & 94 & 112 & 26 & $100.0 \%$ \\
\hline
\end{tabular}

Table 3: Schirmer's test in patients with and without MGD- All the patients without MGD had schirmer's test $>10 \mathrm{~mm}$ but $42(46.7 \%)$ patients with MGD had schirmer's $>10 \mathrm{~mm}, 19(42.2 \%)$ had schirmer's $6-10 \mathrm{~mm}$ and 10 patients $(11.1 \%)$ had schirmer's $<5 \mathrm{~mm}$.

\begin{tabular}{|c|c|c|c|c|}
\hline & \multicolumn{3}{|c|}{ Schirmers } & \multirow{2}{*}{ Total } \\
\hline \multirow{2}{*}{ No MGD } & $\mathbf{1 0 \mathbf { m m }}$ & $\mathbf{6 - 1 0 \mathbf { m m }}$ & $<\mathbf{5 m m}$ & 142 \\
\hline \multirow{2}{*}{ MGD Present } & 142 & 0 & 0 & $100.0 \%$ \\
\cline { 2 - 5 } & $100.0 \%$ & $.0 \%$ & $.0 \%$ & 90 \\
\cline { 2 - 4 } & 42 & 38 & 10 & $100.0 \%$ \\
\hline \multirow{2}{*}{ Total } & $46.7 \%$ & $42.2 \%$ & $11.1 \%$ & 232 \\
\hline & 184 & 38 & 10 & $100.0 \%$ \\
\hline
\end{tabular}

At the end of third month, 34(70.8\%) patients in group A were cured of MGD, and 36(85.7\%) were cured of MGD .

Citation: Venugopal KC, Acharya P, Kavinamoole S, Mundinamani S (2021) Analysis of Effectiveness of Treatment of Meibomian Gland Dysfunction in Patients with Computer Vision Syndrome. J Clin Res Ophthalmol 8(1): 001-006. DOI: https://dx.doi.org/10.17352/2455-1414.000084 
This difference was statisitically significant probably due to inadequate sample size, patient non-compliance with medication, improper lid hygiene.

In group A, by the end of three months, 22 patients(100.0\%) with stage 1 MGD were completely cured, $12(66.7 \%)$ of stage 2 MGD were cured (Table 7) This difference was found to be statistically significant. Total 34 of 48 patients $(70.8 \%)$ were cured of MGD.

In group B, $16(100.0 \%)$ patients with stage 1MGD, 14 $(77.8 \%)$ patients with stage 2 MGD and $6(100.0 \%)$ patients with stage 3 MGD were cured completely at the end of three months $(\mathrm{p}<0.007)$ (Table 8$)$. Total 36 of 42 patients $(85.7 \%)$ were cured of MGD.

\section{Discussion}

Computer Vision Syndrome (CVS), a highly prevalent condition, is frequently associated with dry eye disorders. A reduced blink rate has been observed during computer use [5].

We found significant reduction in blink rate of the patients using VDU for long term. Also tear film breakup time is markedly reduced in patients with history of VDU usage.

Table 4: Blink rate in patients with VDU usage- Among the VDU users $<4 \mathrm{hrs} /$ day $58(34.9 \%)$ had a reduced blink rate $<10 / \mathrm{min}$, but $36(54.5 \%)$ patients who where using VDU $\geq 4 \mathrm{hrs} /$ day had a reduced blink rate.

\begin{tabular}{|c|c|c|c|c|}
\hline & \multicolumn{3}{|c|}{ Blinkrate } & \multirow{2}{*}{ Total } \\
\hline No. Of hours of VDU usage per day & $\mathbf{> 1 5 / m i n}$ & $\mathbf{1 0 - 1 5 / m i n}$ & $<10 / \mathbf{m i n}$ & \\
\hline & 14 & 94 & 58 & 186 \\
\hline <4hours/day & $8.4 \%$ & $56.6 \%$ & $34.9 \%$ & $100.0 \%$ \\
\hline 44 hours/day & 6 & 24 & 36 & 66 \\
\hline & $9.1 \%$ & $36.4 \%$ & $54.5 \%$ & $100.0 \%$ \\
\hline Total & 20 & 118 & 94 & 232 \\
\hline \% within VDUnoofhoursperday & $8.6 \%$ & $50.9 \%$ & $40.5 \%$ & $100.0 \%$ \\
\hline
\end{tabular}

Table 5: Tear film breakup time in patients with VDU usage-12(7.2\%)of VDU users $>4 \mathrm{hrs} /$ day had TBUT $<5$ secs and $14(21.2 \%)$ of VDU users $\geq 4 \mathrm{hrs} /$ day had TBUT $<5$ secs.

\begin{tabular}{|c|c|c|c|c|}
\hline & \multicolumn{3}{|c|}{ TBUT } & \multirow{2}{*}{ Total } \\
\hline No of hours VDU usage perday & & $6-10 \sec$ & $>10$ sec & \\
\hline \multirow{2}{*}{$<4 \mathrm{hrs} /$ day } & 72 & 82 & 12 & 166 \\
\hline & $43.4 \%$ & $49.4 \%$ & $7.2 \%$ & $100.0 \%$ \\
\hline \multirow{2}{*}{$>4$ hrs per day } & 22 & 30 & 14 & 66 \\
\hline & $33.3 \%$ & $45.5 \%$ & $21.2 \%$ & $100.0 \%$ \\
\hline \multirow{2}{*}{ Total } & 94 & 112 & 26 & 232 \\
\hline & $40.5 \%$ & $48.3 \%$ & $11.2 \%$ & $100.0 \%$ \\
\hline
\end{tabular}

Table 6: No. of patients with MGD in group $A$ and $B$. Among this 48 patients in group A, 22 (45.6\%) had stage 1 MGD, 18(37.5\%) patients had stage 2 MGD,8(16.7\%)had stage 3 MGD. Among 42 patients in group B, 16(38.1\%) had stage 1MGD, 18(42.9\%) had stage 2 MGD, $6(14.2 \%)$ had stage 3 MGD, two (4.8\%) patients had stage 4 MGD.

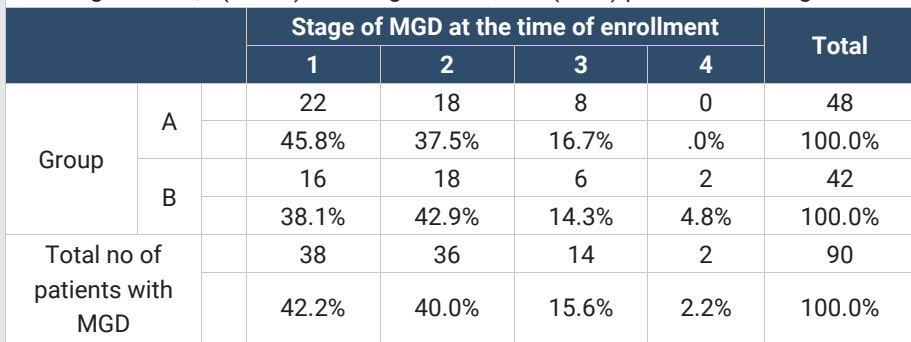

Table 7: No. of patients with MGD in group A cured at the end of three months- 22 patients(100.0\%) with stage 1 MGD were completely cured, $12(66.7 \%)$ of stage 2 MGD were cured.

\begin{tabular}{|c|c|c|c|c|c|c|}
\hline & & \multicolumn{4}{|c|}{$\begin{array}{l}\text { Stage of MGD at the end of three } \\
\text { months }\end{array}$} & \multirow[t]{2}{*}{ Total } \\
\hline & & 0 & 1 & 2 & 3 & \\
\hline \multirow{6}{*}{$\begin{array}{c}\text { Stage of MGD at the time of } \\
\text { enrollment }\end{array}$} & \multirow{2}{*}{1} & 22 & 0 & 0 & 0 & 22 \\
\hline & & $100.0 \%$ & $.0 \%$ & $.0 \%$ & $.0 \%$ & $100.0 \%$ \\
\hline & \multirow{2}{*}{2} & 12 & 6 & 0 & 0 & 18 \\
\hline & & $66.7 \%$ & $33.3 \%$ & $.0 \%$ & $.0 \%$ & $100.0 \%$ \\
\hline & \multirow{2}{*}{3} & 0 & 4 & 2 & 2 & 8 \\
\hline & & $.0 \%$ & $50.0 \%$ & $25.0 \%$ & $25.0 \%$ & $100.0 \%$ \\
\hline \multirow{2}{*}{\multicolumn{2}{|c|}{ Total }} & 34 & 10 & 2 & 2 & 48 \\
\hline & & $70.8 \%$ & $20.8 \%$ & $4.2 \%$ & $4.2 \%$ & $100.0 \%$ \\
\hline
\end{tabular}

Table 8: No. of patients with MGD in group B cured of MGD- 16 (100.0\%) patients with stage 1MGD, 14 (77.8\%) patients with stage 2 MGD and $6(100.0 \%)$ patients with stage 3 MGD were cured completely.

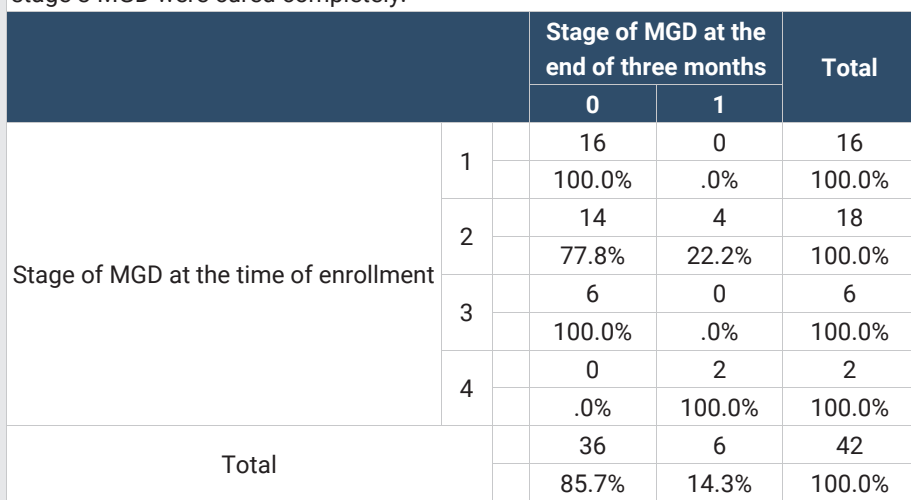

Meibomian gland dysfunction is a chronic and diffuse disorder occurs in meibomian glands, the features are glandular orifices obstruction and/or changes in quality/quantity of the meibomian gland secretion [10]. Many of the computer vision syndrome patients have associated meibomian gland dysfunction due to which symptoms of dry eye aggrevates.

Wang, et al. Study showed a malfunction of meibomian glands is the etiology of dry eye in patients with long term VDU usage [11]. We found significant association of MGD in CVS patients similar to study by Wang, et al. Meibomian gland dysfunction is associated with evaporative dry eye. This increases the symptoms with VDU use.

In patients with computer vision syndrome, dry eye symptoms occur more commonly due to lipid layer insufficiency rather that aqueous deficiency. In long time VDU workers who suffered ocular discomfort and dry eye syndrome without significant aqueous tear volume deficiency, but with significant alterations in meibomian gland morphology and function.

Shimazaki J, et al. study showed $64.6 \%$ patients with ocular discomfort had either an obstruction of an orifice or gland dropout, or both [10]. We found $38.8 \%$ patients with CVS had MGD, where in, 19 patients had stage1 MGD , 18 had stage 2,7 patients had stage 3 and 1 had stage 4 MGD.

In patients with CVS and MGD treated with tear supplements improved in $70.8 \%$ where as when treated with tear supplements along with azithromycin eye ointment, $85.7 \%$ of the patients improved which was statistically significant. 
We also found that $100 \%$ of the CVS patients with stage 1 MGD , $66.7 \%$ of stage 2 MGD and $50 \%$ of the stage 3 MGD improved completely with tear supplements alone where as $25 \%$ of stage 3MGD patients improved to stage 1 and $25 \%$ of stage 3 MGD did not improve with tear supplements alone at the end of three months.

But, $100 \%$ of the CVS patients with stage 1 MGD, $77.8 \%$ of stage 2 MGD, $100 \%$ of stage 3 MGD improved completely and one patient with stage 4 MGD \& $22.2 \%$ stage 2 MGD improved to stage 1 MGD by the end of three months who were treated with tear supplements and azithromycin eye ointment. This difference was statistically and clinically significant.

Hence, all the patient with computer vision syndrome with symptoms of dry eye must be evaluated for features of meibomian gland dysfunction and to be treated. So, treating a computer vision syndrome patient with tear supplements alone will not have a good patient outcome as there is significant association with meibomian gland dysfunction. However, CVS patients with stage 1 and 2 MGD can be treated with tear supplements alone where as a CVS patient with stage 3 and 4 MGD must be treated with azithromycin eye ointment in addition to tear supplements for a better outcome.

\section{Conclusion}

$39 \%$ of CVS patients had Meibomian gland dysfunction. So while treating a computer vision syndrome patient, its also important to evaluate and treat MGD.

Among the CVS patients with MGD, there was a statistically significant improvement in patients $(85.7 \%)$ treated with tear supplements along with azithromycin ointment compared to the patients $(70.8 \%)$ treated with tear supplements alone.

Tear supplements alone can be considered as a treatment option for CVS patients with stage 1 and 2 MGD whereas stage 3 and 4 MGD needs azithromycin eye ointment in addition to tear supplements for a good patient outcome.

\section{References}

1. Read Dry eye chapter in Kanski's Ophthalmology (2016) A systemic Approach; eighth Ed. Elsevier 119-130. Link: https://bit.ly/3ti5w3V

2. Tu YET, Reinstrom S (2014) Dry Eye. In Yanoff N, Duker JS. Ophthalmology Fourth ed. Elsevier.

3. Computer vision syndrome (CVS) (2017) American Optometric Association.

4. Gowrisankaran S, Sheedy JE (2015) Computer vision syndrome: A review. Work 52: 303-314. Link: https://bit.ly/3oER82b

5. Schaumberg DA, Nichols JJ, Papas EB, Tong L, Uchino M, et al. (2011) The international workshop on meibomian gland dysfunction: report of the subcommittee on the epidemiology of, and associated risk factors for MGD. Invest Ophthalmol Vis Sci 52: 1994-2005. Link: https://bit.ly/3oES5Yc

6. Portello JK, Rosenfield M, Chu CA (2013) Blink rate, incomplete blinks and computer vision syndrome. Optom Vis Sci 90: 482-487. Link: https://bit.ly/2YxGhfM

7. Parihar JK, Jain VK, Chaturvedi P, Kaushik J, Jain G, et al. (2016) Computer and visual display terminals (VDT) vision syndrome (CVDTS). Med J Armed Forces India 72: 270-276. Link: https://bit.ly/3ti4Rzt

8. Ranasinghe $P$, Wathurapatha WS, Perera YS, Lamabadusuriya DA, Kulatunga $\mathrm{S}$, et al. (2016) Computer vision syndrome among computer office workers in a developing country: an evaluation of prevalence and risk factors. BMC Res Notes 9: 150. Link: https://bit.ly/3cwQEbX

9. Geerling G, Tauber J, Baudouin C, Goto E, Matsumoto Y, et al. (2011) The international workshop on meibomian gland dysfunction: report of the subcommittee on management and treatment of meibomian gland dysfunction. invest ophthalmol Vis Sci 52: 2050-2064. Link: https://bit.ly/3cyXo9g

10. Shimazaki J, Sakata M, Tsubota K (1995) Ocular surface changes and discomfort in patients with meibomian gland dysfunction. Arch Ophthalmol 113: 1266-1270. Link: https://bit.ly/39EEgVz

11. Wu H, Wang Y, Dong N, Yang F, Lin Z, et al. (2014) Meibomian Gland Dysfunction Determines the Severity of the Dry Eye Conditions in Visual Display Terminal Workers. Plos one 9: e105575. Link: https://bit.ly/36z9sDF

Discover a bigger Impact and Visibility of your article publication with Peertechz Publications

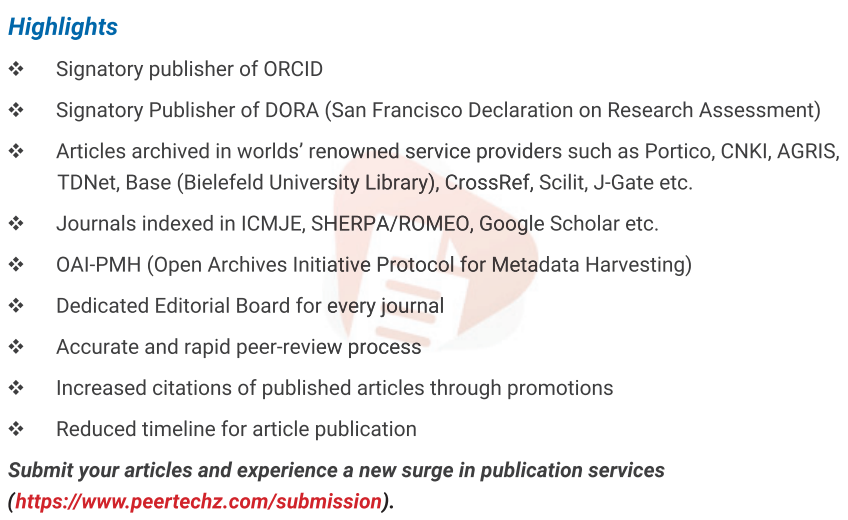

Peertechz journals wishes everlasting success in your every endeavours.

Copyright: @ 2021 Venugopal KC, et al. This is an open-access article distributed under the terms of the Creative Commons Attribution License, which permits unrestricted use, distribution, and reproduction in any medium, provided the original author and source are credited. 